\title{
FOREWORD TO THE SPECIAL ISSUE ON SELECTED PAPERS FROM THE 5TH INTERNATIONAL CONFERENCE ON BIOINFORMATICS AND COMPUTATIONAL BIOLOGY (BICoB 2013)
}

\author{
BHASKAR DASGUPTA \\ University of Illinois at Chicago \\ HISHAM AL-MUBAID \\ University of Houston-Clear Lake \\ FAHAD SAEED \\ National Institutes of Health
}

Published 22 August 2013

\begin{abstract}
With the rapid increase in the volume of biological data generated using next generation technologies, designing algorithms to process these data in an efficient manner and gaining biological insight is becoming a significantly growing challenge. This special issue is a follow-up to the 5th International Conference on Bioinformatics and Computational Biology (BICoB 2013) that took place in Honolulu, Hawaii during March 4-6, 2013. The guest editors selected a few top-quality papers from the $\mathrm{BICoB}$ proceedings and invited the authors of these papers to submit extended versions of their proceedings papers. After a rigorous review process the guest editors have selected the following five papers described below for publication in this special issue.

The first paper titled "Studying the role of APOE in Alzheimer's disease (AD) pathogenesis using a systems biology model" ${ }^{1}$ presents one of the first attempts to model AD from a systems approach to study physiologically relevant parameters that may prove useful in the future.

The second paper titled "A hybrid-system model of the coagulation cascade: simulation, sensitivity, and validation" ${ }^{2}$ presents a model of the blood coagulation cascade as a hybrid system (HS) consisting of interacting continuous and discrete dynamics. Extensive simulations of the model provide a platform for in silico investigation of blood clotting.
\end{abstract}


The third paper is titled "Dynamic Bayesian clustering" 3 and introduces a Bayesian clustering approach that is able to formulate dynamic clusters which split and merge over time, in order to detect time-dependent groupings.

The fourth paper titled "Exact solutions for species tree inference from discordant gene trees" 4 presents a dynamic programming solution to gene tree parsimony (GTP) and RF supertree problem. Interesting results are reported for real and simulated data sets and comparisons are performed between different algorithms.

The fifth and last paper titled "CNCTDiscriminator: Coding and non-coding transcript discriminator - an excursion through hypothesis learning and Ensemble learning approaches" ${ }^{5}$ presents a collection of models trained with different perspectives of features of transcripts, where each model is capable of discriminating two classes of transcripts. The paper also introduced a feature-specific Ensemble approach in building a specialized model which outperforms prediction performances of those models that are built only with the individual category of features.

We would like to thank all the authors that submitted papers to this special issue. Our special thanks go to all the reviewers who unselfishly devoted their professional time to assist us in our decision process.

\section{References}

1. Kyrtsos CR, Baras JS, Studying the role of APOE in Alzheimer's disease pathogenesis using a systems biology model, J Bioinform Comput Biol 11(5):1342003, 2013.

2. Makin JG, Narayanan S, A hybrid-system model of the coagulation cascade: Simulation, sensitivity, and validation, J Bioinform Comput Biol 11(5):1342004, 2013.

3. Fowler A, Menon V, Heard NA, Dynamic Bayesian clustering, J Bioinform Comput Biol 11(5):1342001, 2013.

4. Chang W-C, Gorecki P, Eulenstein O, Exact solutions for species tree inference from discordant gene trees, J Bioinform Comput Biol 11(5):1342005, 2013.

5. Biswas AH, Zhang B, Wu X, Gao JX, CNCTDiscriminator: Coding and non-coding transcript discriminator - an excursion through hypothesis learning and Ensemble learning approaches, J Bioinform Comput Biol 11(5):1342002, 2013. 